\title{
Buchbesprechung von: Połońska, Eva/Beckett, Charlie: Public service broadcasting and media systems in troubled European democracies
}

\author{
Schweizer, Corinne
}

Abstract: Połońska und Beckett, beide ehemalige Journalisten und heute an der London School of Economics (LSE) angestellt, stellen folgende Frage: Können traditionelle Medien, die nach westlich-liberalem Vorbild operieren und daraus auch ihre normative Rechtfertigung ziehen - als Stichworte nennen sie Vielfalt, informierte und aufgeklärte Bürger, Wachhund der Demokratie - das öffentliche Interesse gegenüber dem Staat auch in diesem neuen, post-demokratischen Umfeld sichern?

DOI: https://doi.org/10.1007/s11616-019-00544-9

Posted at the Zurich Open Repository and Archive, University of Zurich

ZORA URL: https://doi.org/10.5167/uzh-179261

Journal Article

Accepted Version

Originally published at:

Schweizer, Corinne (2020). Buchbesprechung von: Połońska, Eva/Beckett, Charlie: Public service broadcasting and media systems in troubled European democracies. Publizistik, 65(1):107-109.

DOI: https://doi.org/10.1007/s11616-019-00544-9 


\section{Public Service Broadcasting and Media Systems in troubled European Democracies}

\section{Besprechung von Corinne Schweizer}

Vor 2015 hat Bogusława Dobek-Ostrowska Polen noch dem 'hybrid-liberalen' Medienmodell zugeordnet. Denn wie die baltischen Staaten, Slowenien, Slowakei und Tschechien war Polen unter den ehemals kommunistischen Zentral- und Osteuropäischen Staaten ein Spitzenreiter der wirtschaftlichen Entwicklung, Demokratisierung und Medienfreiheit. Seit 2015 die PiS-Partei (deutsch: Recht und Gerechtigkeit) an die Macht kam, teilt Dobek-Ostrowska Polen jedoch wie Bulgarien, Kroatien, Ungarn, Rumänien und Serbien dem 'politisierten' Modell zu. In all diesen Ländern fehlt es an Medienfreiheit, dafür blüht der Klientelismus. Die Entwicklung in Polen rief 2016 die Europäische Union auf den Plan. Zum ersten Mal setzte man hier einen neuen medienpolitischen Hebel an: Wegen Verstosses gegen das fundamentale Recht der Meinungs- und Informationsfreiheit drohte die EU damit, Polens Mitgliedschaftsrechte einzuschränken. Die Drohung wirkte, schreibt Carles Llorens: PiS änderte die umstrittenen neuen Regeln für den öffentlichen Rundfunk unverzüglich.

Polen ist zwar nur eines von vielen Europäischen Ländern bzw. Mediensystemen, die im Sammelband 'Public Service Broadcasting and Media Systems in troubled European Democracies' von Eva Połońska und Charlie Beckett beleuchtet werden. Ihm kommt im Buch jedoch eine wichtige Bedeutung zu. Die Medienpolitik der PiS wird nicht nur von Dobek-Ostrowksa's und Llorens angesprochen, sie wird auch in zwei weiteren Fallstudien von Stanisław Mocek und von Połońska selbst untersucht. Vermutlich ist der Fokus auf Polen der Teilfinanzierung des Buches durch das 'Programm für ein modernes Polen' der an der Universität Oxford situierten Noble Stiftung geschuldet. Er lässt sich aber auch inhaltlich sehr gut begründen: Polen verkörpert - gemeinsam mit Ungarn - am deutlichsten die von Połońska und Beckett in vielen Ländern Europas beobachtete oder zumindest befürchtete Erosion liberaldemokratischer Ideale und den Trend hin zu einer 'neuen Illiberalität'.

Połońska und Beckett, beide ehemalige Journalisten und heute an der London School of Economics (LSE) angestellt, stellen folgende Frage: Können traditionelle Medien, die nach westlich-liberalem Vorbild operieren und daraus auch ihre normative Rechtfertigung ziehen - als Stichworte nennen sie Vielfalt, informierte und aufgeklärte Bürger, Wachhund der Demokratie - das öffentliche Interesse gegenüber dem Staat auch in diesem neuen, post-demokratischen Umfeld sichern? Um darauf Antworten zu finden, führten Połońska und Beckett im Dezember 2016 an der LSE einen Workshop durch, zu dem sie Forschende aus Ost-, Süd- und Westeuropa einluden und aus dem dieses Buch entstand. Wenige Wochen vor dem Workshop war der US-Präsident Donald Trump gewählt worden und im Juni 2016 hatte das britische Volk für den Brexit gestimmt. Geprägt von diesen Eindrücken lauschten die westeuropäischen Forschenden den Erzählungen ihrer Kolleginnen und Kollegen aus Süd- und Osteuropa an diesem Workshop mit grosser Aufmerksamkeit, als diese von den illiberalen medienpolitischen Praktiken in ihren Ländern berichteten.

Der im Titel verwendete vieldeutige Begriff 'troubled' ist eine klug gewählte semantische Klammer, um die nach wie vor grossen Unterschiede zwischen den Ländern Europas einzufangen. Der Begriff kann 'unruhig', 'besorgniserregend' oder gar 'gestört' bedeuten. Die Situation in den ersten beiden Fallstudien zu Frankreich und Belgien dürfte im Vergleich eher in die Kategorie 'unruhig' fallen. In Italien, Griechenland, Spanien und den Balkanstaaten können sich die Medien hingegen bis heute der politischen Einflussnahme nicht entziehen. Ähnlich 'besorgniserregend' sieht die Situation in Polen aus. Die eigentlichen 'troubled democracies', daraus machen Połońska und Beckett keinen Hehl, befinden sich jedoch in Europas 'Peripherie': In Ungarn, Bulgarien und Rumänien, der Ukraine, Russland und der Türkei. Diese Länder und ihre Medien, das zeigen die entsprechenden Kapitel 
deutlich, befinden sich in einer 'Grauzonen zwischen Demokratie und Autoritarismus'; ihr öffentlicher Rundfunk ist ein unfreier Staatsrundfunk.

Gemäss Połońska und Beckett richtet sich ihr Buch an Personen, die sich mit politischer Kommunikation, Demokratie, und Populismus beschäftigen. Diese demokratietheoretische Ausrichtung macht es zu einer guten Ergänzung zur populären Sammelbandreihe zum öffentlichen Rundfunk 'Ripe' (Re-Visionary Interpretations of the Public Enterprise) die alle zwei Jahre im NordicomVerlag erscheint. Die Ripe-Bücher blenden politische Aspekte zwar keineswegs aus, sind jedoch aus einer Medienmanagement-Perspektive geschrieben. Połońska und Beckett erfüllen grundsätzlich auch den eigenen Anspruch, einen 'aktuellen Überblick' zu geben. Gerade Studierenden oder Forschenden, die sich mit Europäischen Mediensystemen im Rahmen ihrer politischen Systeme vertraut machen wollen, bieten die Kapitel ein idealer Einstieg. Mit den traditionellen Medien wohlgemerkt, denn der Einfluss der sozialen Medien oder von Algorithmen steht hier nicht im Zentrum.

Was dem Sammelband jedoch - wie vielen anderen seiner Art - fehlt, ist ein abschliessendes, vergleichendes Kapitel, das die einleitend gestellte Frage noch einmal aufnimmt und eine Antwort, einen Ausblick und mögliche Lösungsoptionen formuliert. Dass der öffentliche Rundfunk nach wie vor die Medieninstitution ist, die dem liberal-demokratischen Ideal am meisten entspricht, wird durch das einleitende Kapitel von Stephen Cushion zu Medieninhalten und -effekten gleich vorweggenommen. Doch was ist zu tun, wenn der öffentliche Rundfunk wegen des politischen Einflusses in vielen europäischen Ländern nicht so funktioniert, wie er sollte? Interessanterweise formuliert das bereits erwähnte, ebenfalls einleitende Kapitel von Llorens zur erstarkten medienpolitischen Rolle der Europäischen Union einige ganz konkrete Lösungsoptionen.

Diese Version der Buchbesprechung wurde von 'Publizistik' zur Veröffentlichung akzeptiert. Die finale Publikation ist unter https://link.springer.com/article/10.1007/s11616-019-00544-9 zugänglich.

This is the version of a book review accepted for publication by 'Publizistik'. The final publication is available at https://link.springer.com/article/10.1007/s11616-019-00544-9. 